\title{
Diced and grounded broadband bow-tie antenna with tuning stub for resonant tunnelling diode terahertz oscillators
}

\author{
ISSN 1751-8725 \\ Received on 1st June 2016 \\ Revised 6th September 2016 \\ Accepted on 23rd September 2016 \\ E-First on 6th December 2016 \\ doi: 10.1049/iet-map.2016.0395 \\ www.ietdl.org
}

\author{
Khalid Hamed Alharbi ${ }^{\bowtie}$, Ata Khalid ${ }^{1}$, Afesomeh Ofiare ${ }^{1}$, Jue Wang ${ }^{1}$, Edward Wasige ${ }^{1}$ \\ ${ }^{1}$ High Frequency Electronics Group, School of Engineering, University of Glasgow, UK \\ 凶E-mail: k.alharbi.1@research.gla.ac.uk
}

\begin{abstract}
Radiation from antennas integrated with indium phosphide (InP)-based resonant tunnelling diode (RTD) oscillators is mainly through the substrate because of the effects of the large dielectric constant. Therefore, hemispherical lenses are used to extract the signal from the backside of the substrate. In this study the authors present a broadband bow-tie slot antenna with a tuning stub which is diced and mounted on a ground plane to alleviate the substrate effects. Here, the large dielectric constant substrate around the antenna conductor is removed. In addition, the ground plane underneath the diced substrate acts as a reflector and, ultimately, the antenna radiates to the air-side direction. Antenna integration with RTD oscillators is described in this study as well. Two-port bow-tie slot antennas were designed and characterised and showed the suitability of integration with power combining RTD oscillator circuits which are based on mutual coupling. The antennas were fabricated using electron beam lithography on a semi-insulating InP substrate. Simulated and measured bandwidth almost extends the entire frequency range $230-325 \mathrm{GHz}$. Simulations shows air-side radiation pattern and antenna gain of around $11 \mathrm{~dB}$ at $280 \mathrm{GHz}$. Simulations also show that the antenna may be fed with a $50-\Omega$ or $30-\Omega$ feed line, i.e. suitable feed lines, without compromising its performance which may prove beneficial for optimum loading of RTD oscillators.
\end{abstract}

\section{Introduction}

Terahertz $(\mathrm{THz})$ radiation, whose frequency range lies between millimetre-waves and infrared light in the electromagnetic spectrum has many potential applications in different scientific fields such as medical diagnostics, security imaging, and wireless communication [1-4]. Indium phosphide (InP) based resonant tunnelling diode (RTD) $\mathrm{THz}$ oscillators in integrated circuit (IC) form have recently been shown to have the capability of producing $\mathrm{THz}$ frequencies at room temperature with relatively high power [5-9]. However, there are a number of challenges when designing antennas on large dielectric constant, $\varepsilon_{r}$, substrate such as InP substrate $\left(\varepsilon_{r}=12.56\right)$. Antennas on such substrates tend to radiate most of their energy into and through the substrate [10]. For example, an infinitesimal planar dipole radiates approximately $\varepsilon_{r}^{3 / 2}$ more power into the substrate than into free space. In addition, any radiation into the substrate at angles greater than the critical angle $\left(\theta_{\mathrm{c}}=\sin ^{-1}\left(\varepsilon_{r}^{-1 / 2}\right)\right)$ are totally internally reflected at the top and bottom surfaces and leading to confinement of the energy inside

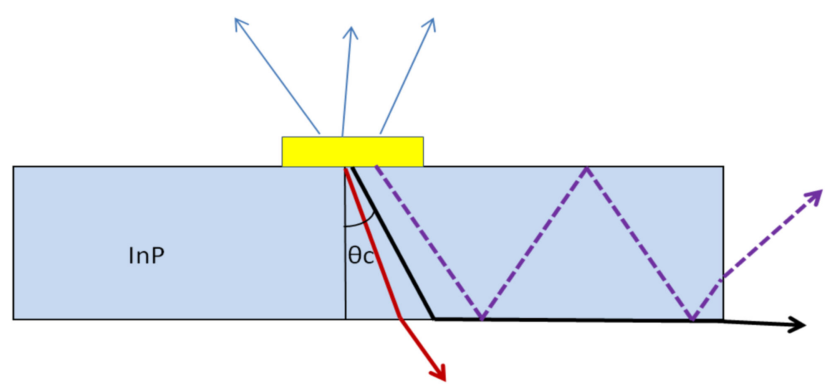

Fig. 1 Illustration of the critical angle and radiation of antenna fabricated on large dielectric constant substrate. Any radiation with angle greater than the critical angle $\left(\theta_{\mathrm{c}}=\sin ^{-1}\left(\varepsilon_{r}^{-1 / 2}\right)\right)$ will be totally internally reflected at the top and bottom surfaces and leading to confinement of the energy inside the substrate. This confined radiation propagates inside the substrate without getting radiated unless a discontinuity appears at the edges. Moreover, most of the energy is directed into the substrate side compared with the air-side the substrate as illustrated in Fig. 1. For InP, the critical angle is around $16.4^{\circ}$. This confined radiation propagates inside the substrate without getting radiated unless a discontinuity appears at the edges leading to degradation in the radiation pattern and antenna performance in general. Usually, a hemispherical lens is used to collect the power from the back side of the substrate [11]. In this paper, instead of using bulky lenses, we propose a broadband bow-tie slot antenna with a tuning stub which is diced and mounted on a ground plane for InP-based RTD oscillators. By limiting the substrate area around the antenna conductor, the substrate effects can be reduced. Furthermore, the ground plane underneath the die acts as reflector to re-direct the energy to the air-side region of the antenna.

To date, RTD oscillators have used slot antennas which, besides directing most of the radiation through the substrate, have other limitations such as the narrow bandwidth. Slot antennas integrated with RTD oscillators have been used in short range communications at $542 \mathrm{GHz}$ with a range of only $\sim 1 \mathrm{~cm}[12]$. Another reported antenna type for RTD oscillators is the patch antenna with benzocyclobutene (BCB) used as the substrate and isolated from the InP substrate [13]. However, it is only feasible beyond $750 \mathrm{GHz}$ (i.e. can employ relatively thin BCB substrate at higher frequencies for acceptable performance) and it is narrowband. An antenna with high gain and directivity is preferable in order to radiate most of the RF power into a specific direction and offer larger distance coverage.

The rest of the paper is organised as follows. Section 2 presents the bow-tie slot antenna used in the proposed concept. The effects of the substrate size are analysed and discussed. The advantages of the proposed diced and grounded antenna are presented in this section. Section 3 presents the simulation and measurement results of the proposed antenna. Section 4 describes the potential of integrating the bow-tie slot antenna with RTD oscillators. It also presents characterisation results for a two port bow-tie slot antenna which also can be integrated with power combining oscillator circuits based on mutual coupling between oscillators. Conclusions and future work are provided in Section 5. 


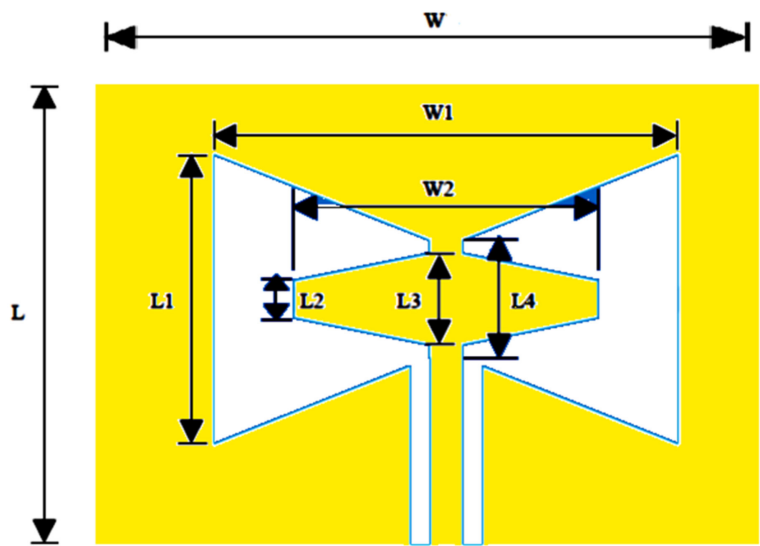

Fig. 2 Geometry of a bow-tie slot antenna with a tuning stub [14]

\section{Bow-tie slot antenna with tuning stub}

\subsection{Antenna design}

A bow-tie slot antenna is a simple version of planar slot antennas which can offer large bandwidth. A simple coplanar waveguide (CPW) fed bow-tie slot antenna with a tuning stub for increased bandwidth adopted in this work was proposed in [14]. Fig. 2 shows the geometry of the design. For a desired bandwidth, the antenna dimensions are calculated as a function of the free space wavelengths at the lower frequency $\left(\lambda_{\mathrm{L}}\right)$ and the higher frequency $\left(\lambda_{\mathrm{H}}\right)$, where $W_{1}=0.77 \lambda_{\mathrm{H}}, W_{2}=0.40 \lambda_{\mathrm{L}}, L_{1}=0.33 \lambda_{\mathrm{L}}, L_{2}=0.03 \lambda_{\mathrm{L}}, L_{3}$ $=0.09 \lambda_{\mathrm{H}}, L_{4}=0.10 \lambda_{\mathrm{H}}, W=1.3 \times W_{1}$ and $L=1.12 \times L_{1}$. In this paper the antenna was designed for operation between 200 and $350 \mathrm{GHz}$ with the following antenna dimensions: $W_{1}=660 \mu \mathrm{m}, W_{2}=600$ $\mu \mathrm{m}, L_{1}=495 \mu \mathrm{m}, L_{2}=45 \mu \mathrm{m}, L_{3}=77 \mu \mathrm{m}, L_{4}=96 \mu \mathrm{m}, W=840$ $\mu \mathrm{m}$, and $L=556.5 \mu \mathrm{m}$. The stubs were employed in the design to increase the input resistance, which results in better matching.
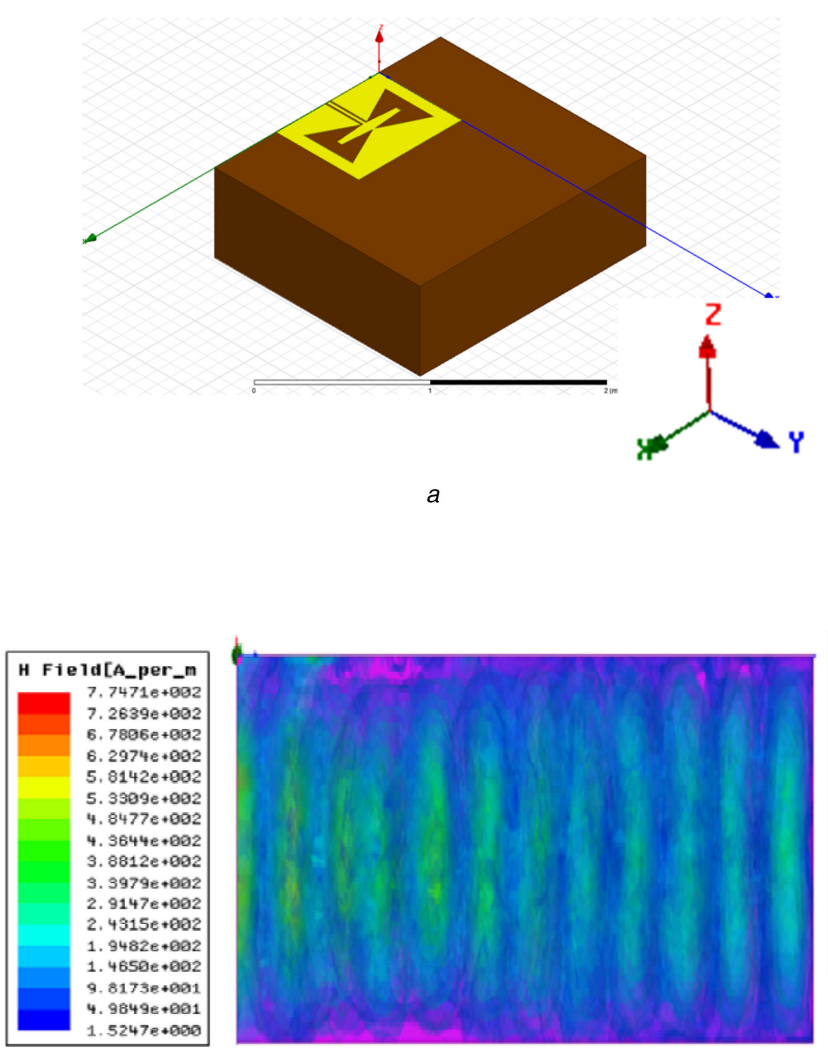

Moreover, the stubs shift the main resonance to a lower frequency and create a new resonance at a higher frequency [14]. The antenna bandwidth can be further optimised by changing the design parameters.

Two identical antennas, one with a $50-\Omega \mathrm{CPW}$ feed line and the other with a $30-\Omega$ CPW feed line were investigated. This was done to compare the antenna bandwidth in the two cases since the input impedance of a bow-tie slot antenna seems to assume that of the feeding line (at least for the known $50-\Omega$ case) and also since the antenna has been designed mainly for integration with RTD devices which may have different negative differential conductance values and therefore can directly affect the impedance matching to the antenna load. The signal line width of $20 \mu \mathrm{m}$ and gap of 13.8 $\mu \mathrm{m}$ to the ground planes were calculated to give $50 \Omega \mathrm{CPW}$ feed line, while $20 \mu \mathrm{m}$ signal line width and $2.5 \mu \mathrm{m}$ gap were used to give $30 \Omega \mathrm{CPW}$ feed line. The metallisation was made of $0.4 \mu \mathrm{m}$ thick gold and patterned on the top of the InP substrate.

\subsection{Effects of the large dielectric constant substrate}

As discussed above, for $\operatorname{InP}\left(\varepsilon_{r}=12.56\right)$ the critical angle $\theta_{\mathrm{c}}$ is $16.4^{\circ}$. Therefore, any radiation into the substrate at angles greater than this angle will be confined inside the substrate. To visualise the effect of the substrate on the radiated energy, the bow-tie slot antenna was simulated on a large area InP (Fig. $3 a$ ) using HFSS simulator. Figs. $3 b$ and $c$ show the electric and magnetic fields inside the substrate, respectively. These fields confirm the inevitable degradation of the antenna performance when using a planar antenna on a large area substrate of large dielectric constant. The radiation pattern is shown in Fig. $3 d$. It can be seen that the radiation pattern is unpredictable with no clear lobe that indicates the antenna directivity.

\subsection{Diced and grounded antenna}

The effects of the substrate can be alleviated if the substrate material around the antenna conductors is removed. This makes the
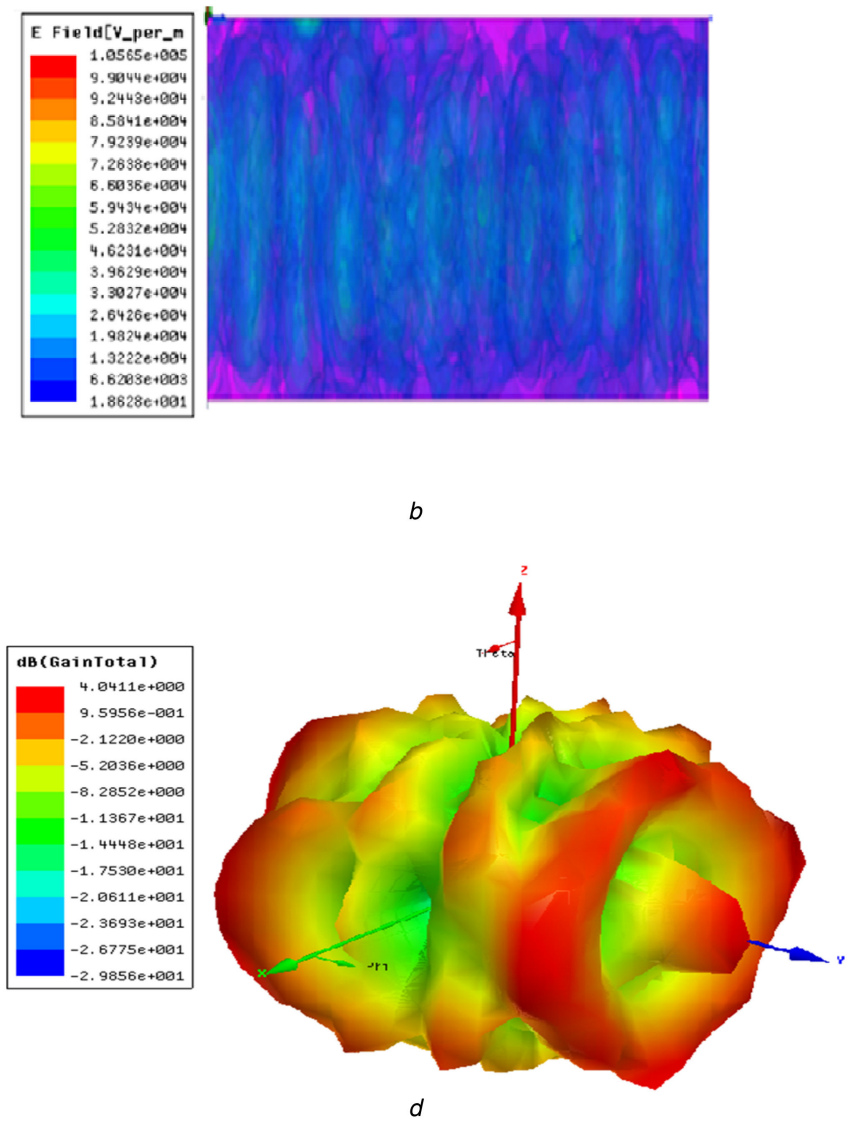

Fig. 3 Investigations of the effects of the size of the InP substrate on the antenna performance

(a) Antenna geometry on a relatively large area substrate compared with the antenna dimensions, (b) E-field inside the substrate (side view, $y z$-plane), (c) H-field inside the substrate (side view, $y z$-plane), (d) Radiation pattern of the bow-tie slot antenna placed on a large area InP substrate. The radiation has no clear pattern 


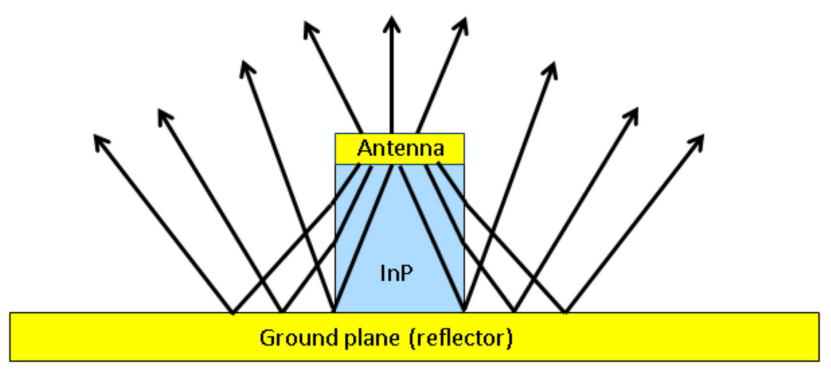

Fig. 4 Illustration of the radiation of a diced antenna placed on reflector ground plane. The substrate material around the antenna is removed to alleviate substrate effects. The antenna chip is mounted on a large ground plane which reflects radiation directed into the substrate back upwards so that the overall antenna radiation is directed to the air-side of the antenna rather than to the substrate side

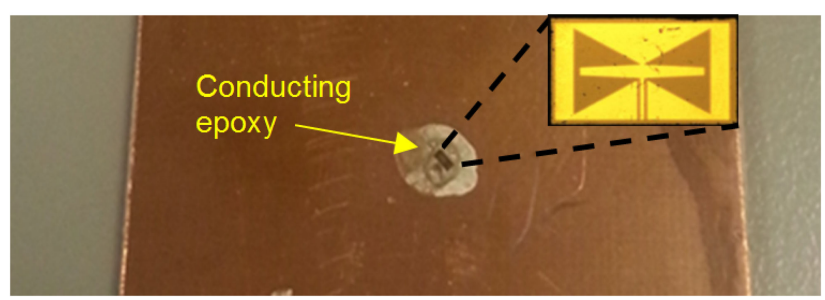

Fig. 5 Fabricated diced bow-tie slot antenna placed on a reflector ground plane. The inset shows a micrograph of the actual antenna

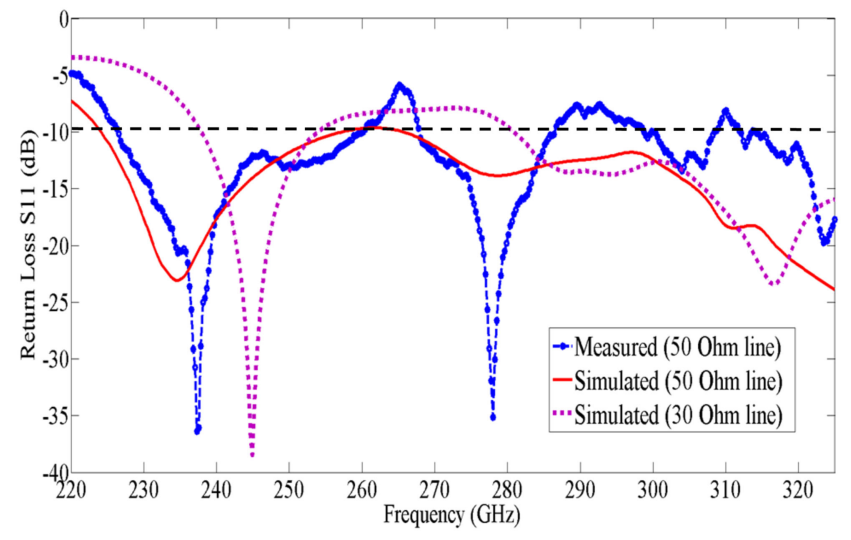

a into the substrate back upwards as illustrated in Fig. 4. Ultimately, therefore, the diced and grounded antenna radiates towards its airside region.

The bow-tie slot antenna with a tuning stub was fabricated using electron beam lithography. Electron beam resist polymethyl methacrylate (PMMA) was used. The spun PMMA on the InP sample was baked for $3 \mathrm{~min}$ at $160^{\circ} \mathrm{C}$, followed by e-beam pattern writing using a Vestec VB6 beam writer. The exposed PMMA was then developed in a $1: 1$ mixture of methyl isobutyl ketone (MIBK) and isopropyl alcohol, followed by deposition of a Ti/Au $(20 \mathrm{~nm} / 400 \mathrm{~nm})$ metal scheme using an electron beam evaporator and lift-off in acetone. The antenna was then diced and mounted on a large reflector ground plane. Fig. 5 shows a picture of the fabricated antenna mounted with conducting epoxy on a ground/ metallic plane.

\section{Results}

\subsection{Return loss, antenna efficiency and radiation pattern}

The return loss of the fabricated antenna shown in Fig. 5 was measured using a J-band $(220-325 \mathrm{GHz})$ Cascade Microtech millimetre wave vector network analyser (VNA) with groundsignal-ground (G-S-G) infinity probes. The VNA was calibrated for 1-port S-parameter measurements using the short-open-load one port calibration process on a Cascade impedance substrate. Fig. $6 a$ shows the measured and simulated return loss of the designed antenna with a $50 \Omega$ characteristic impedance CPW feed line. The bandwidth, defined by return loss of less than $-10 \mathrm{~dB}$, extends almost the entire frequency band from $220-325 \mathrm{GHz}$. The

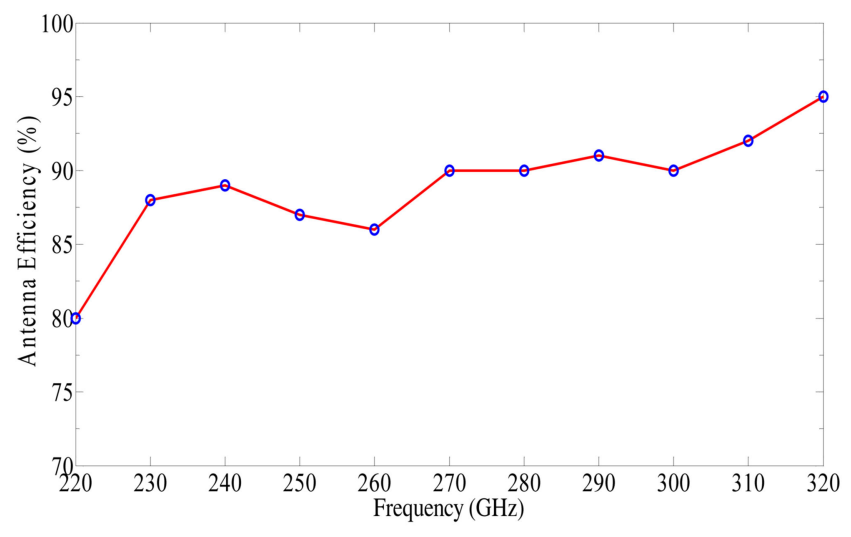

$b$

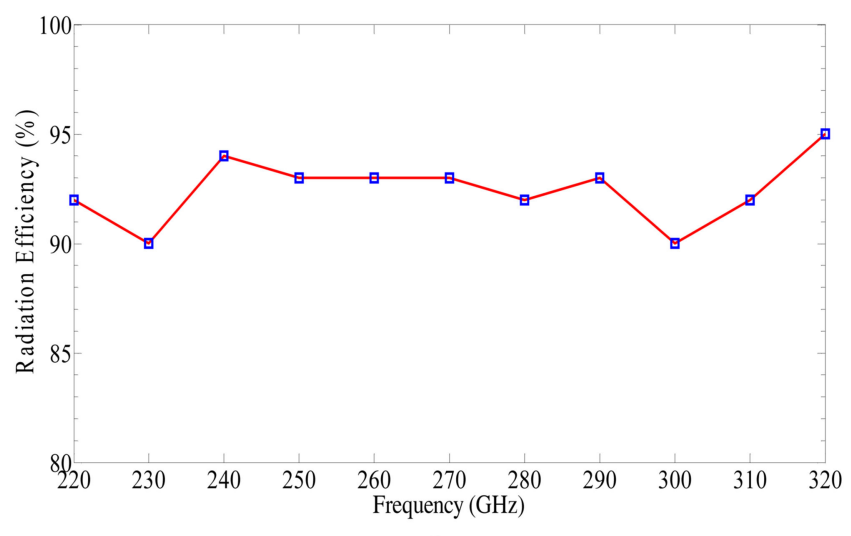

$c$

Fig. 6 Return loss and efficiency of the one port bow-tie slot antenna

(a) Simulated (with 50 and $30 \Omega$ feed lines) and measured return loss (with $50 \Omega$ feed line). The antenna with a $50 \Omega$ feed line demonstrates broadband performance across the entire J-band and the antenna with $30 \Omega$ feed line shows comparable bandwidth, (b) Simulated overall efficiency of the one port bow-tie slot antenna which describes the ratio of the radiated power to the incident power, (c) Simulated radiation efficiency of the one port bow-tie slot antenna which describes the ratio of the radiated power to the accepted (delivered) power

size of the substrate the same as the outer dimensions of the antenna. Therefore, the path of confined radiation will be shorter. Furthermore, placing this diced antenna on a large reflector ground plane will enhance the radiation pattern by re-directing radiation discrepancy between the measured and simulated results can be attributed to unwanted signal reflections from surrounding objects during measurements, the short CPW lengths underneath the probes, etc. The return loss of the antenna with a $30 \Omega \mathrm{CPW}$ feed 

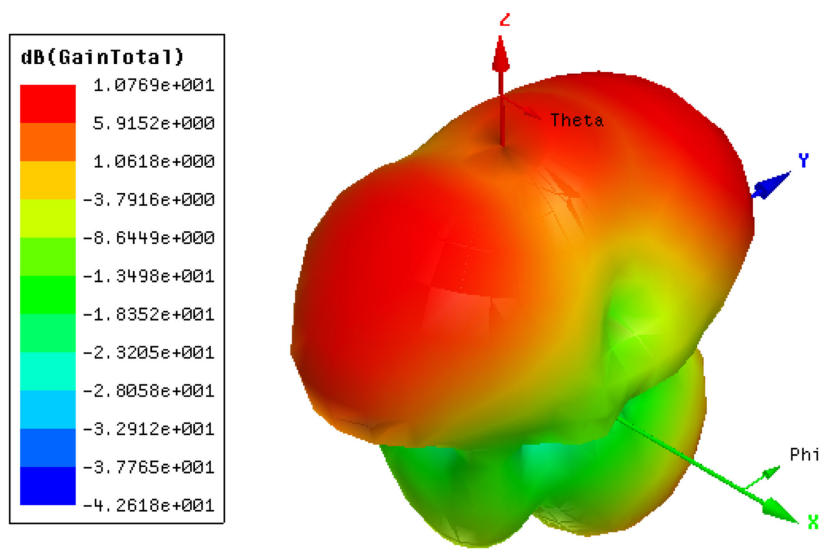

Fig. 7 Simulated $3 D$ radiation pattern of the one port bow-tie slot antenna at $280 \mathrm{GHz}$. It has a maximum gain of $11 \mathrm{~dB}$ and radiates upwards toward the air-side region of the antenna

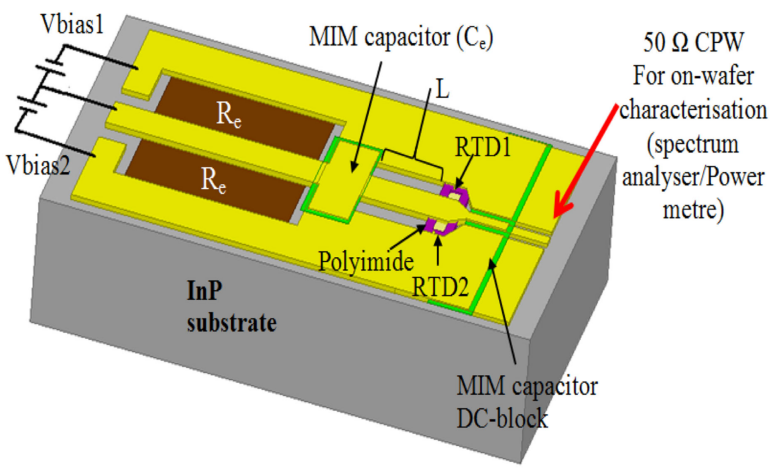

a the antenna at $280 \mathrm{GHz}$ is shown in Fig. 7. The antenna maximum gain is around $11 \mathrm{~dB}$ and the proposed antenna radiates upwards toward the air-side direction.

\section{Antenna integration with RTD-based oscillators}

\subsection{Integration with single RTD oscillator}

A power combining RTD-based oscillator circuit was proposed and demonstrated by the authors in $[5,15,16]$. In this configuration, the oscillator circuit employs two RTD devices connected in parallel and delivers power to a single load. Fig. $8 a$ shows an illustration of this power combining RTD oscillator circuit. Recently, such RTD oscillators with oscillation frequencies in the $245-310 \mathrm{GHz}$ range and output powers from $0.2-0.5 \mathrm{~mW}$ have been demonstrated and the results will be presented elsewhere [17]. The oscillators are designed for on-wafer characterisation using a spectrum analyser to detect the oscillation frequencies and a power meter to measure the oscillator output power. The spectrum analyser has an input impedance of $50 \Omega$ and so on-wafer

Fig. 8 Integrating the bow-tie slot antenna with the power combining RTD-based oscillator

(a) Schematic structure for the two RTDs oscillator. Details on the design and different components can be found in [5, 15, 16], (b) Schematic structure for integrating the bow-tie slot antenna presented in this paper with the two RTD oscillator

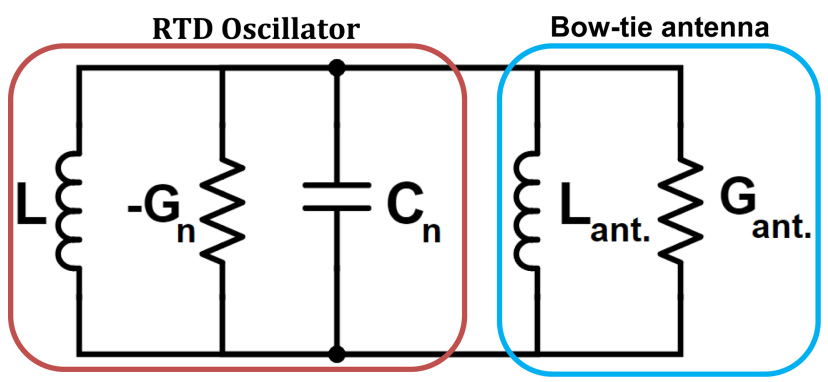

Fig. 9 Small-signal equivalent circuit of an RTD oscillator integrated with bow-tie slot antenna. $L$ denotes the resonating inductance realised in the RTD oscillator circuit. $-G n$ and $C n$ are the RTD device negative differential conductance and the self-capacitance, respectively. $L_{\text {ant. }}$ and $G_{\text {ant }}$ are the inductance and the conductance of the integrated bow-tie slot antenna, respectively

line was also simulated and shown in Fig. $6 a$. The bandwidth of the antenna is comparable with that of the antenna with a $50 \Omega \mathrm{CPW}$ feed line. Therefore, the feed line can be chosen according to the RTD's negative differential conductance to improve the impedance matching and so deliver higher power.

The simulated antenna overall efficiency (defined as the ratio of the radiated power to the incident power) and the antenna radiation efficiency (defined as the ratio of the radiated power to the accepted (delivered) power) are plotted in Figs. $6 b$ and $c$, respectively. The antenna overall efficiency is $80 \%$ at the lowest frequency in the band $(220 \mathrm{GHz})$ and increases to $95 \%$ at $320 \mathrm{GHz}$. The radiation efficiency is around $92 \%$ on average across the entire simulated frequency band. The simulation software (HFSS) takes into accounts all losses (e.g. in the feed line) in the antenna structure when it calculates the radiation efficiency and does not assume a lossless structure. The simulated $3 \mathrm{D}$ radiation pattern of characterisations are carried out using G-S-G RF probes landed on the $50 \Omega \mathrm{CPW}$ transmission line pads as shown in Fig. $8 a$. Since the bow-tie slot antenna presented in this paper is a CPW fed, it would be practically suitable for integration with this oscillator layout. Fig. $8 b$ shows a proposed schematic structure for this RTD oscillator-antenna integration in which two RTD devices are located close to the centre of the bow-tie slot antenna. The metalinsulator-metal capacitors (DC-block) in Fig. $8 b$ are placed to isolate the two electrodes of the bow-tie slot antenna and act as a short circuit at the designed frequency.

Fig. 9 shows the small-signal equivalent circuit of an RTD oscillator integrated with a bow-tie slot antenna load. The antenna reactance could change the oscillation frequency of the RTD oscillator and its effect can be explained by means of this equivalent circuit which includes the resonating inductance $L$ in parallel with $-G_{n}$ and $C_{n}$ which are the RTD device negative differential conductance and the self-capacitance, respectively. The bow-tie slot antenna is represented by $L_{\text {ant. }}$ and $G_{\text {ant. }}$ which are its self-inductance and the (radiation) conductance, respectively. The equivalent oscillator inductance is, therefore, $L_{\text {eq }}=L_{\text {ant }} L /\left(L_{\text {ant. }}+L\right)$. Hence, if the antenna inductance $L_{\text {ant. }}$ is much larger than the resonating inductance $L$ then the oscillation frequency will not change. The simulated reactance part of the antenna is plotted in Fig. $10 a$. The reactance part of the antenna is inductive across the frequency range from 220 to $308 \mathrm{GHz}$ and the equivalent inductances are plotted in Fig. 10b. At some frequencies, the antenna inductance is much higher than the resonating inductance $L$ (which is typically in the range of 2 to 4 $\mathrm{pH}$ ) and so no significant modification in the oscillation frequency is expected. The real part of the antenna input impedance is shown in Fig. $10 c$. In the $270-325 \mathrm{GHz}$ range, it is approximately $50 \Omega$. 

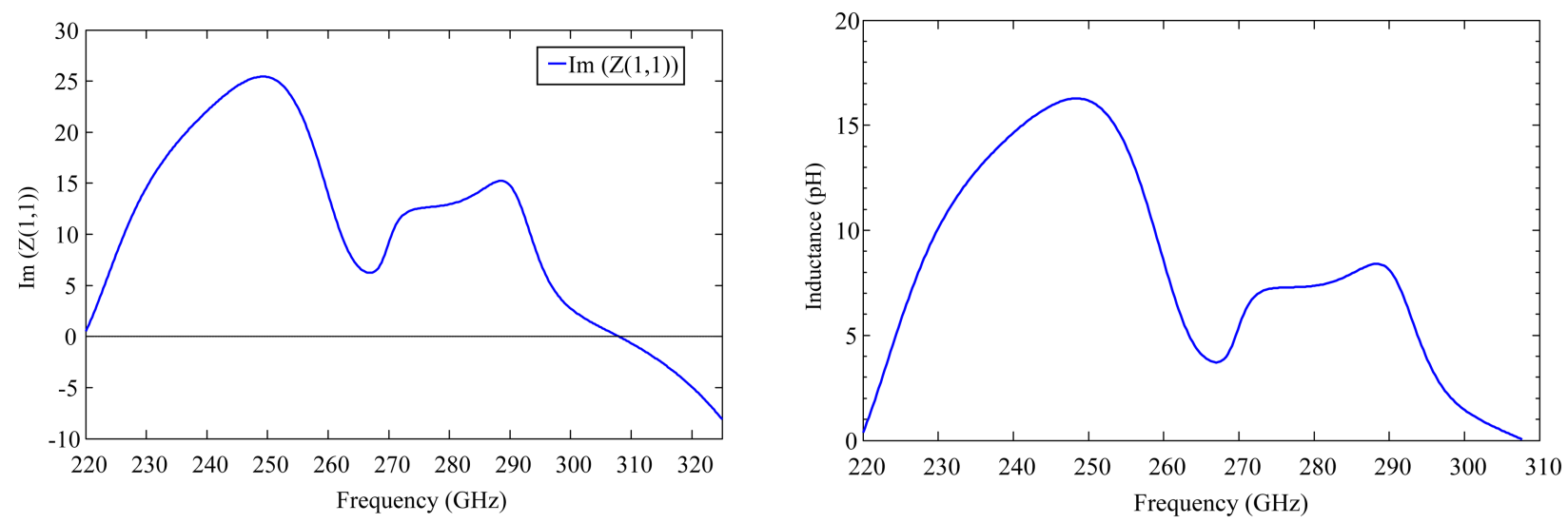

a

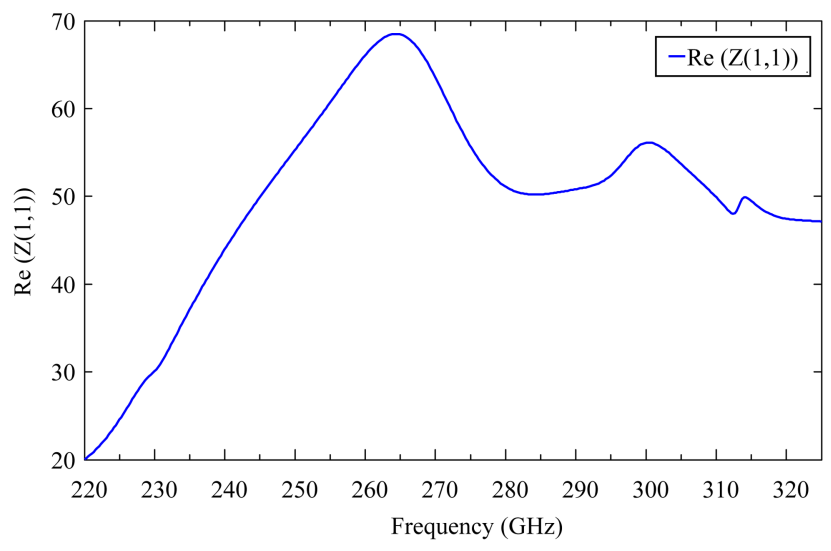

Fig. 10 Simulated reactance (imaginary part) and resistance (real part) of the input impedance of the bow-tie slot antenna with $50 \Omega$ CPW feed line (a) Simulated reactance showing the antenna is inductive across the frequency range from $220-308 \mathrm{GHz}$, (b) Extracted equivalent inductances. At some frequencies, the antenna inductance is much higher than the resonating inductance of the RTD oscillator circuit (which is usually in the range of 2 to $4 \mathrm{pH}$ ) and so no significant modification in the oscillation frequency is expected, (c) Simulated input resistance of the bow-tie slot antenna. It is approximately $50 \Omega$ from $270-325 \mathrm{GHz}$

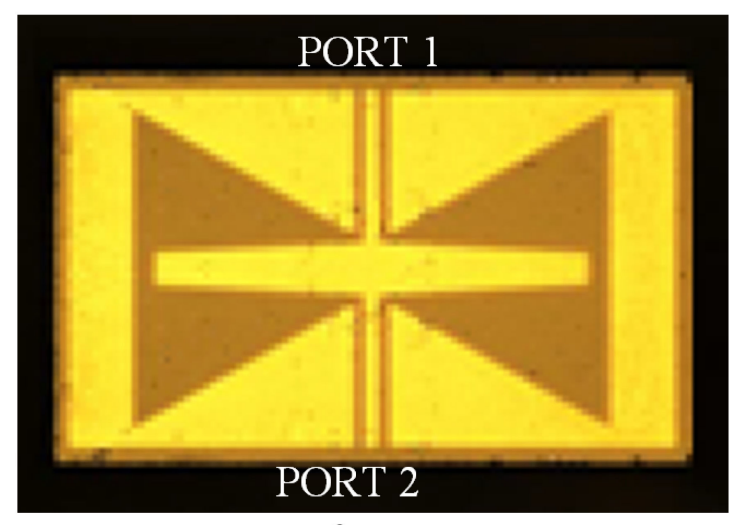

a
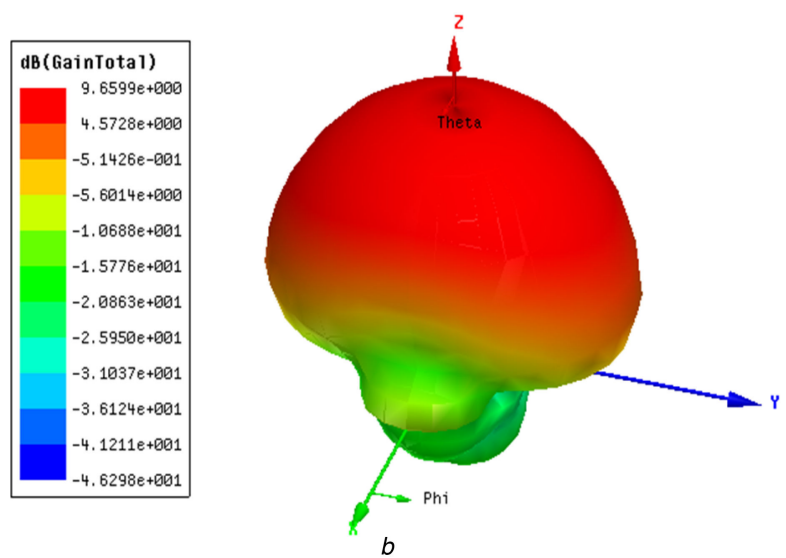

Fig. 11 Two port bow-tie slot antenna with two CPW feed lines

(a) Fabricated bow-tie slot antenna with two CPW feed lines where port 1 and port 2 are used to characterise the antenna, (b) Simulated radiation pattern at 280 GHz. The antenna has a maximum gain of $9.7 \mathrm{~dB}$

\subsection{Two-port bow-tie slot antenna: integration with mutually coupled RTD oscillators}

Another power combining RTD oscillator circuit which can employ four RTD devices and deliver higher output power to a single load has been proposed by the authors recently [18]. It couples the output power of two oscillators (shown in Fig. 8a) into a single load. Using this topology, practically relevant output power of $1.1 \mathrm{~mW}$ at $\sim 310 \mathrm{GHz}$ was obtained from an IC realisation and results will be published elsewhere soon. As this load could be an antenna, the bow-tie slot antenna presented in this paper was further investigated with a two port bow-tie slot antenna design. The antenna was designed with the same dimensions presented above but with two $50 \Omega \mathrm{CPW}$ feed lines. Fig. $11 \mathrm{a}$ shows the fabricated two port bow-tie slot antenna, while Fig. $11 b$ shows the simulated radiation pattern at $280 \mathrm{GHz}$.

The simulated and measured return loss $\left(S_{11}\right.$ and $\left.S_{22}\right)$ of the two port bow-tie slot antenna are shown in Fig. 12a, while Fig. $12 b$ shows the simulated and measured insertion loss $\left(S_{21}\right.$ and $\left.S_{12}\right)$. All the four S-parameters indicate that most of the signal send towards the antenna is accepted, and little is reflected back $\left(S_{11}, S_{22}<-10\right.$ $\mathrm{dB})$ or transmitted $\left(S_{21}, S_{12}<-10 \mathrm{~dB}\right)$ through to the other port. Compared with the return loss obtained from the one port antenna in Fig. $6 a$, the return loss from the two ports are similar in general with some discrepancy that might be due to the measurement environment, e.g. close proximity of the pair of probes and reflections. The simulated antenna overall efficiency and the 

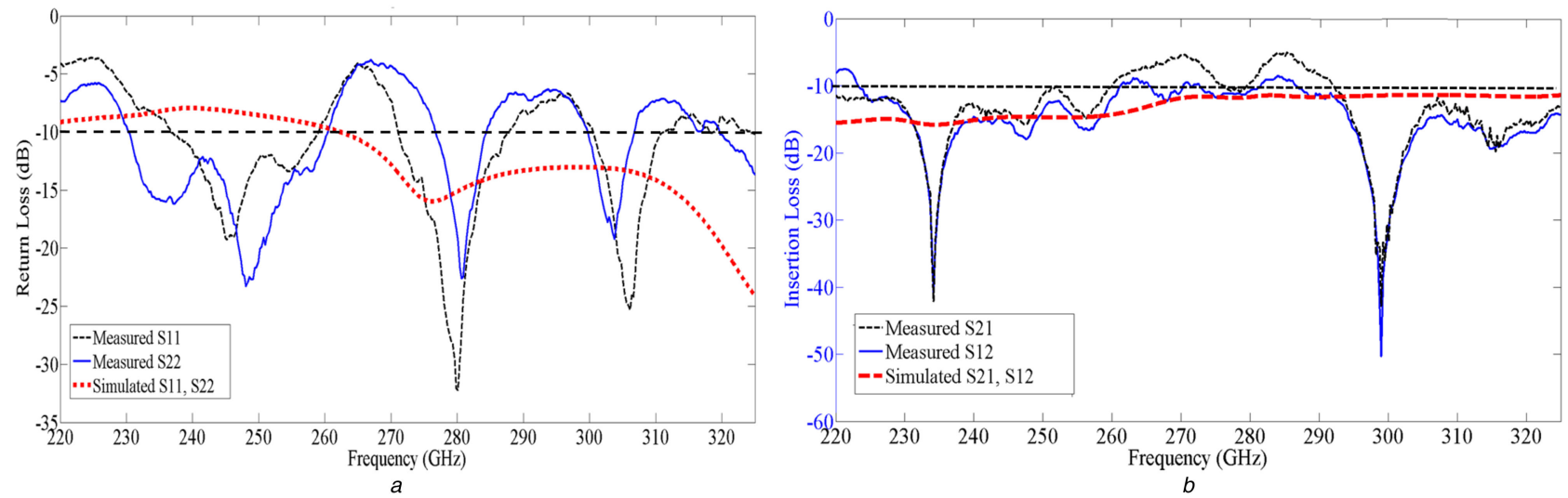

Fig. 12 Simulated and measured results for the two port bow-tie slot antenna with tuning stubs

(a) Simulated and measured return loss $\left(S_{11}\right.$ and $\left.S_{22}\right)$ of the two port bow-tie slot antenna with stubs. The results indicate that the signal send towards the antenna is not reflected back; return loss less than $-10 \mathrm{~dB}$ e.g. in the $230-260 \mathrm{GHz}$ range, etc., (b) Simulated and measured insertion loss $\left(S_{21}\right.$ and $\left.S_{12}\right)$ of the two port bow-tie slot antenna with stubs. The results indicate that little of the signal send towards the antenna is transmitted through the other port. Therefore together, all the four S-parameters show that the antenna radiates the energy fed into its input terminals
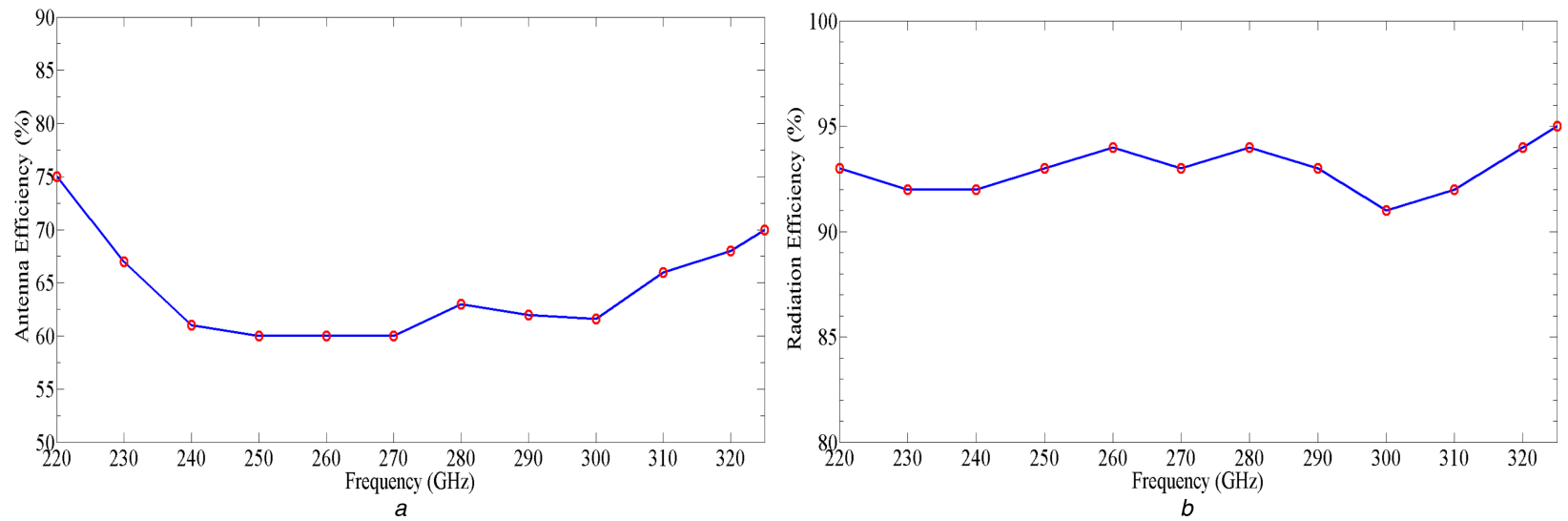

Fig. 13 Simulated efficiencies of the two port bow-tie slot antenna

(a) Simulated overall efficiency of the two port bow-tie slot antenna which describes the ratio of the radiated power to the incident power, (b) Simulated radiation efficiency of the two port bow-tie slot antenna which describes the ratio of the radiated power to the accepted (delivered) power

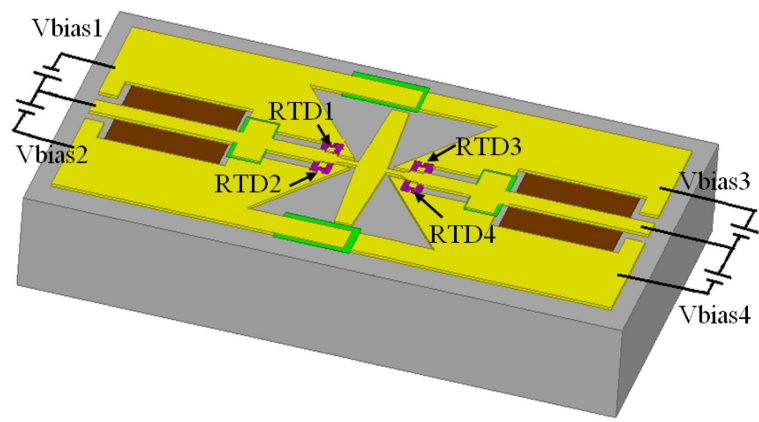

Fig. 14 Schematic structure for integrating the two port bow-tie slot antenna with the coupled RTD oscillators

antenna radiation efficiency of the two port bow-tie slot antenna are plotted in Figs. $13 a$ and $b$, respectively. The antenna overall efficiency is $65 \%$ on average while the radiation efficiency is around $93 \%$ on average across the entire simulated frequency band. Thus, the two port bow-tie slot antenna is a good candidate to be integrated with the coupled oscillator circuit that employs four RTD devices for high power operation. Fig. 14 shows possible schematic structure of this integration.

\section{Conclusions}

In this paper, a grounded and diced bow-tie slot antenna with a tuning stub for terahertz applications was presented. It offers a known input impedance and wide bandwidth. The diced and grounded antenna suppresses substrate effects and radiates towards its air-side region. The performance of the one port and two port bow-tie slot antennas was investigated. Results showed that these antennas are suitable for integration with different power combining RTD oscillator circuits. Future work is focused on integrating antennas with InP-based RTD THz oscillators.

\section{Acknowledgments}

The authors thank the staff of the James Watt Nanofabrication Centre (JWNC) at the University of Glasgow for help in fabricating devices. This work was supported in part by the Engineering and Physical Sciences Research Council (EPSRC) of the UK, grant number EP/J019747/1, and in part by the European Commission, grant agreement no. 645369 (iBROW project).

\section{References}

[1] Siegel, P.H.: 'Terahertz technology', IEEE Trans. Microw. Theory Tech., 2002, 50, (3), pp. 910-928

[2] Tonouchi, M.: 'Cutting-edge terahertz technology', Nat. Photonics, 2007, 1, pp. $97-105$

[3] Kleine-Ostmann, T., Nagatsuma, T.: 'A review on terahertz communications research', J. Infrared Millim. Terahertz Waves, 2011, 32, pp. 143-171

[4] Liu, H.B., Zhon, H., Karpowicz, N., et al.: 'Terahertz spectroscopy and imaging for defense and security applications', Proc. IEEE, 2007, 95, pp. $1514-1527$

[5] Wang, J., Alharbi, K., Ofiare, A., et al.: 'High performance resonant tunneling diode oscillators for $\mathrm{THz}$ applications'. IEEE Compound Semiconductor Integrated Circuit Symp. (CSICS), New Orleans, LA, USA, 11-14 October 2015, pp. 1-4

[6] Maekawa, T., Kanaya, H., Suzuki, S., et al.: 'Frequency increase in terahertz oscillation of resonant tunnelling diode up to $1.55 \mathrm{THz}$ by reduced slotantenna length', Electron. Lett., 2014, 50, (17), pp. 1214-1216

[7] Suzuki, S., Hanashima, K., Kishimoto, N., et al: 'Sub-THz resonan tunneling diode oscillators with offset-fed slot antenna'. Int. Conf. on Indium Phosphide and Related Materials, Matsue, Japan, 2007, pp. 530-533 
[8] Hinata, K., Shiraishi, M., Suzuki, S., et al:: 'High power THz oscillators with offset-fed slot antenna and high current density resonant tunneling diodes'. Int. Conf. on Infrared, Millimeter, and Terahertz Waves, Busan, 2009, pp. 1-2

[9] Suzuki, S., Hinata, K., Shiraishi, M., et al.: 'RTD oscillators at $430-460 \mathrm{GHz}$ with high output power $(\sim 200 \mu \mathrm{W})$ using integrated offset slot antennas'. Int. Conf. on Indium Phosphide Related Materials, Kagawa, 2010

[10] Brown, E.R., Parker, C.D., Yablonovitch, E.: 'Radiation properties of a planar antenna on a photonic-crystal substrate', J. of the Opt. Soc. Am. B, 1993, 10, pp. 404-407

[11] Orihashi, N., Suzuki, S., Asada, M.: 'One THz harmonic oscillation of resonant tunneling diodes', Appl. Phys. Lett., 2005, 87, pp. 233501-1233501-3

[12] Ishigaki, K., Shiraishi, M., Suzuki, S., et al.: 'Direct intensity modulation and wireless data transmission characteristics of terahertz-oscillating resonant tunnelling diodes', Electron. Lett., 2012, 48, (10), pp. 582-583

[13] Koyama, Y., Sekiguchi, R., Ouchi, T.: 'Oscillations up to $1.40 \mathrm{THz}$ from resonant-tunneling-diode-based oscillators with integrated patch antennas', Appl. Phys. Express, 2013, 6, (6), p. 064102
[14] Eldek, A.A., Elsherbeni, A.Z., Smith, C.E.: 'Characteristics of bow-tie slot antenna with tapered tuning stubs for wideband operation', Progr Electromagn. Res., 2004, 49, pp. 53-69

[15] Wang, J., Wang, L., Li, C., et al.: ' $28 \mathrm{GHz}$ MMIC resonant tunneling diode oscillator of around $1 \mathrm{~mW}$ output power', Electron. Lett., 2013, 49, (13), pp. 816-818

[16] Wang, J., Wang, L., Li, C., et al.: 'W-band InP-based resonant tunnelling diode oscillator with milliwatt output power'. The 26th Int. Conf. on Indium Phosphide and Related Materials, Montpellier, France, May 2014

[17] Wang, J., Al-Khalidi, A., Alharbi, K., et al.: 'High performance resonant tunnelling diode oscillators as terahertz sources'. European Microwave Conf., London, October 2016

[18] Ofiare, A., Wang, J., Alharbi, K., et al.: 'Novel tunnel diode oscillator power combining circuit topology based on synchronisation'. Asia-Pacific Microwave Conf. (APMC), Nanjing, 2015, pp. 1-3 